\title{
Attacks toward Wireless Network-on-Chip and Countermeasures
}

\author{
Arnab Kumar Biswas, Navonil Chatterjee, Hemanta Kumar Mondal, \\ Guy Gogniat and Jean-Philippe Diguet \\ Lab-STICC, CNRS / Université de Bretagne Sud, Lorient, France 56100
}

Final submission to IEEE TETC Feb. 6, 2020.

\section{Introduction}

A Wireless Network-on-Chip (WiNoC) offers a promising solution to reduce broadcast and long distance communication bottlenecks of conventional architectures by augmenting them with single hop wireless links. In this paper, we discuss new security vulnerabilities and countermeasures to protect against them in a WiNoC based system. In particular, we describe Malicious Threshold Configuration (MTC) Attack, Disruptive Token Passing (DTP) Attack, Data Stealing by Broadcast (DSB) Attack and Hybrid Attack against the WiNoC. Our proposed countermeasure against MTC-OU (over-utilization) attack i.e., Source Destination checking mechanism decreases wireless hub utilization by $49 \%$ and network latency by many orders of magnitude compared to without countermeasure, causing system performance improvement. Another proposed countermeasure against DTP attacks i.e., detour mechanism improves the network throughout by $1.21 x$ and $23 x$ under DTP-AHT and DTP-DOS attacks respectively. Keywords:Wireless Network-on-Chip, Attack, Countermeasure.

\section{Introduction}

Network-on-Chip (NoC) is a well known on-chip communication medium that is used to communicate packets between different processing elements inside a chip. Recent advances in Multiprocessor Systemon-Chip (MPSoC) design and their corresponding increase in practical use cases call for strong security guarantees. NoC is an important component of any massively parallel MPSoC system and hence a secure NoC contributes to the overall system security.

Parallel applications running in high performance computing servers get performance benefits due to the use of NoCs in the server MPSoC chips. The primary components of parallel applications are cache coherency and synchronization. Both of them face significant challenges during broadcast or multi-cast operations due to critical path communications. Conventional NoC architectures support broadcast operations in the form of multiple uni-cast transmissions, which results in significant system performance penalties concerning network latency and energy consumption overheads. A Wireless Network-on-Chip (WiNoC) offers a promising solution to reduce the long distance or critical path communication bottle- necks of conventional NoC architectures by augmenting them with single hop, long-range wireless links. NoC security has been studied for years [1] but these wireless links introduce new security risks into the system.

As per our understanding, only few works $[2,3,4]$ exist in literature that analyze the threats against WiNoC. Even in these existing works the details of attacks and their effects on the system are not discussed. We want to fill that gap by providing details of attacks and their effects on the system which clearly shows the need of security consideration while designing such systems. In this paper, we describe three new types of attacks and a hybrid attack specific to a WiNoC based system: Malicious Threshold Configuration (MTC) Attack, Disruptive Token Passing (DTP) Attack, Data Stealing by Broadcast (DSB) Attack and Hybrid Attack. We also provide the countermeasures against these attacks and a comparison with the existing works.

The main contributions of this work are as follows:

1. We introduce three new attacks i.e., MTC, DTP and DSB attacks in a WiNoC system. Among them, MTC and DTP have 2 sub-types. Also a hybrid attack is introduced which results in thermal attack.

2. We evaluate these attacks on a real WiNoC system and show their effects with respect to different parameters like latency, packet loss, throughput etc.

3. We propose countermeasures to protect from these new attacks.

4. We provide simulation results in presence of our countermeasures clearly showing their effectiveness against the attacks.

The rest of the paper is organized as follows. In Section 2, existing WiNoC works are discussed including those that do not consider security. Next, the system architecture and the threat model considered in this paper are described in Section 3. Section 4 provides the description of our proposed attacks and also provides the simulation results showing their adverse effects on the system. Next, the countermeasures to the proposed attacks are given in Section 5 and their effectiveness are also shown through simulation results. Section 6 concludes the paper. 


\section{Related Works}

In this Section we discuss WiNoC based works that do not consider security before discussing the works that consider security threats against WiNoC.

\subsection{WiNoC without security}

Works exist in literature that aim to maximize the utilization of WiNoC targeting various WiNoC architectures, topologies and routing methods. Authors in [5] have proposed and evaluated the performance of various WiNoC architectures and [6] has proposed a hybrid wired and wireless network architecture. A recursive, WiNoC structure called the WCube is proposed in [7] that features a single transmit antenna and multiple receive antennas at each micro wireless router. Authors in [8] have proposed the design of a smallworld WiNoC architecture with mm-wave wireless interconnects used as long-range links and have shown in $[9,10]$ that the smallworld WiNoC outperforms wired NoC. A hybrid WiNoC architecture called iWISE and a honeycomb-based WiNoC architecture called $H^{2} \mathrm{WNoC}$ are proposed in [11] and [12] respectively. Authors in [13] have presented the design of an adaptive CDMA protocol for WiNoC and a geo-assisted routing scheme for irregular mesh WiNoCs is proposed in [14]. An Adaptive Multi-Voltage Scaling (AMS) method to reduce router power consumption in WiNoC is proposed in [15]. In [16], authors have used WiNoC routers to implement a directory based cache coherence mechanism to minimize communication latency in a multicore architecture.

Works also exist in literature that model the wireless communication channel to simulate and to evaluate the WiNoC platform better and also to improve the network design. In [17], authors have proposed a multi-channel WiNoC platform and in [18], a communication channel in the $\mathrm{Ka}$ band is proposed to improve the on-chip communication of many-core chips. A parameterizable wireless channel model is proposed in [19] to evaluate the losses in WiNoC and to simulate the platform better. In [20], authors have shown the impact of a semi-realistic multipath wireless channel over conventional WiNoC modulation scheme and have proposed a digital transceiver architecture. A survey of the modeling of wireless channel for WiNoC is presented in [21].

Apart from the WiNoC architectures and channel models, works are also targeted to design efficient transceivers which allow the wireless signal transmission and reception. An energy efficient mm-wave transceiver at $65 \mathrm{~nm}$ CMOS technology is proposed in [22] and an OFDM transceiver that is robust against channel effects and provides high data rate is proposed in [23]. Authors in [24] have proposed an adaptive digital transceiver using channel compensation techniques for single and multiple parallel channel access modes. In [25], authors have presented a reliability aware runtime tunable transmitting power technique for improving the energy efficiency of the transceiver. In [26], a low-power high-speed OOK de- modulator in $60-\mathrm{GHz}$ band is presented. A survey of various design possibilities and challenges for WiNoC architectures is presented in [27] where security is not taken into consideration.

\subsection{WiNoC with security}

Security consideration in $\mathrm{NoC}$ is a domain of high research interest which deals with different types of attacks and countermeasures.

Authors in [2] consider a WiNoC architecture where each core has its own wireless component to send and receive packets apart from the wired router connection. They consider both contention free method like token passing protocol and contention based method like carrier sense multiple access (CSMA) protocol. In CSMA, each node can transmit at any time giving flexibility but collisions can occur. At the network interface, they collect network performance statistics to implement congestion avoidance and security policy.

They assume that Hardware Trojan (HT) is responsible for attacks but it is not clear how it can create packets or its location. It's only known that it's not present in the physical layer (PHY) that is responsible for transmission and reception of bits over wireless links. In our work, we consider contention free token passing method, which is common in most existing WiNoC works. We provide clear description of the node architecture (regular router or hub router) that is under attack and also show the various adverse effects on the system.

The protection method presented in [2] assumes that the node will send a packet to the OS to notify about attack. Next, the OS can block the wireless interface temporarily or permanently. If this is applied to a system where more than one router is connected to one wireless transmitter, the protection method itself can be used to block (DoS attack) the applications running in the co-located processors which are connected to the other regular routers.

Authors in [3] have targeted a 64-core system that implements a cache coherency protocol called ECONO. They assume 16 antennas and transceivers at each $\mathrm{L} 2$ receivers. There are 16 L3 senders and 64 L2 receivers in the system. Their assumption of receiver setup is non-realistic. But it allows all to all congestion free communication. They use a cryptographic hash function called SPONGENT to provide integrity that requires 450 clock cycles to compute the hash value. They load a counter (to protect from replay attack) and key value from memory to L2 cache directly during page table walk. Then these are sent from L2 to L3 cache via NoC wired links. Their proposed hash based solution requires more than $30 \%$ performance overhead which is not practical. In this scenario any solution which gives better performance is desirable.

Authors in [4] consider DoS attack due to presence of $\mathrm{HT}$ in a core which triggers garbage packets into the router. The packets will create congestion in the network from source to the neighbouring routers. Their proposal is to form a new NoC topology that will have better resistance against DoS traffic. They 
use the smallworld network topology for this purpose where connection between nodes is dependent on the distance and the frequency of traffic interaction between the two cores. That means the connections are application dependent and not generic. They have also applied Simulated Annealing heuristic to optimize the network in order to minimize the spreading of DoS attack. Their solution can only minimize the DoS attack effect but cannot stop it. Also the derived topology is application dependent and any other application will need a different topology.

From the above description of related works, it is clear that there is a scope of further analysis of WiNoC under different attacks. In this work, in addition to such analysis under various novel attacks, we also provide various countermeasures to prevent such attacks.

\section{System Architecture threat model}

and

In this section we discuss the system architecture that we are considering in this work and also provide the threat model against this system along with the attack mechanism.

\subsection{System Architecture}

We consider a $8 \times 8$ WiNoC with four equally-sized clusters as shown in Figure 1(a). Each cluster consists of 16 nodes which are arranged in $4 \times 4$ fashion. Every cluster has a centrally placed wireless hub with a wireless interface (WI) providing inter-cluster wireless links. There are two types of routers in the WiNoC: normal routers that are connected to the processing elements (PEs) and hub routers that are connected to the wireless interfaces. Both types of routers perform uni-cast and broadcast communication. At the source router, a decision is made regarding use of wireless link for communication. Depending on the decision, packets can flow only through the normal routers or use both normal routers and wireless hubs. For broadcast operation, the hubs are always used.

We use Time Division Multiple Access (TDMA) based token passing technique which divides token holding time for different hubs into time slots. For a given time slot, the hub which has the token will be able to transmit packet to other hub. Please note that other hubs which do not have the token will be able to receive the transmitted packet. This policy helps to avoid collision between different hubs. In our work, the packets first arrive at a hub to be transmitted but they may need to wait for the availability of the token. Once the hub is ready to transmit, it sends the packets. Currently we do not use any acknowledgment to send packets from a hub. So if a packet is lost during wireless transmission, the sender hub does not know about this. Please note that we use the term hub to indicate hub router containing the wireless transceiver. Further details of router architecture including hub router architecture are given in next section.

\subsection{Normal Router architecture}

We consider a 5-port router in our system architecture, which consists of North, South, East, West, and Local ports, respectively. Figure 1(b) shows a normal router architecture. For ease of understanding, we only show a single input and output port. The routers are configurable and they are configured before the start of normal operation. The Configuration Module receives configuration packets from all input ports except the local port and configures Threshold Configuration Register (TCR) of every input port of the router. When a normal packet arrives at the input FIFO, the Decision Logic (DL) decides if the packet must be routed through the wireless hub or through wired communication based on the TCR. Depending on the decision, it sends a request signal to the corresponding output port through the Routing Logic Unit (RLU) and later sends the packet when the output port is available. The RLU is responsible for routing packets for both wired and wireless communication. In case of wireless communication, the packets are routed to the hub router for wireless transmission.

We call the routers which are directly connected to the hub as hub connection (HC) router. These HC routers have a sixth port which is exclusively used to connect to the hub. That means in some routers, the Request Generator for output Port module has five out going signals instead of four as shown in Figure $1(\mathrm{~b})$.

\subsubsection{Routing Algorithm}

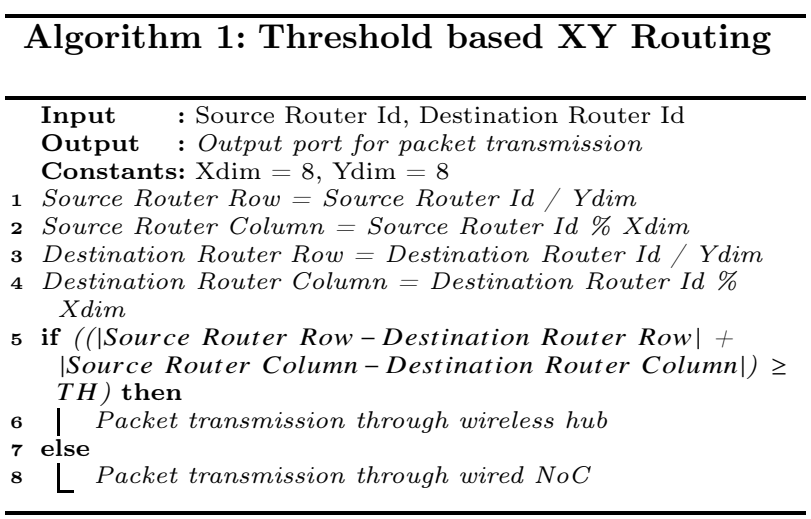

To route packets from source to destination node, we have used a threshold based XY routing algorithm similar to [28]. We assume that every router knows the topological information, such as size of the network (X and $\mathrm{Y}$ dimension) and number of wireless Nodes. The head flit in the packet contains the source and destination address. First, the manhattan distance between the source and destination nodes is calculated. Algorithm 1 presents the threshold based $\mathrm{XY}$ routing strategy. If the manhattan distance between the source and destination nodes is greater than a given threshold then the packet is routed using the wireless hub. Alternatively, it is routed through wired NoC. To route a packet through wireless hub, first it is routed to the $\mathrm{HC}$ router which sends it to the hub router. The source hub transmits the packet to its 


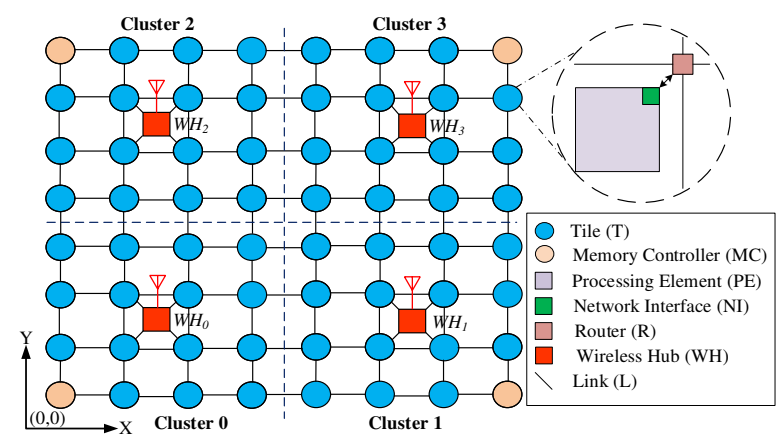

(a)

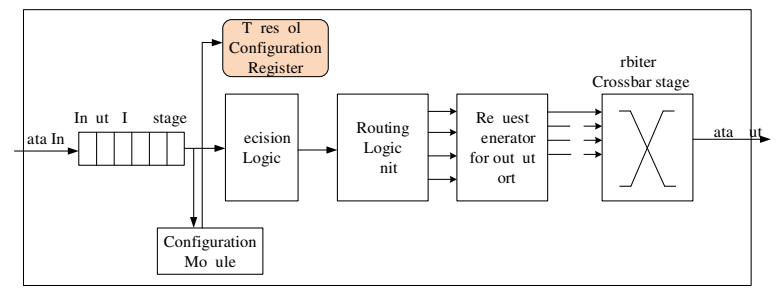

(b)

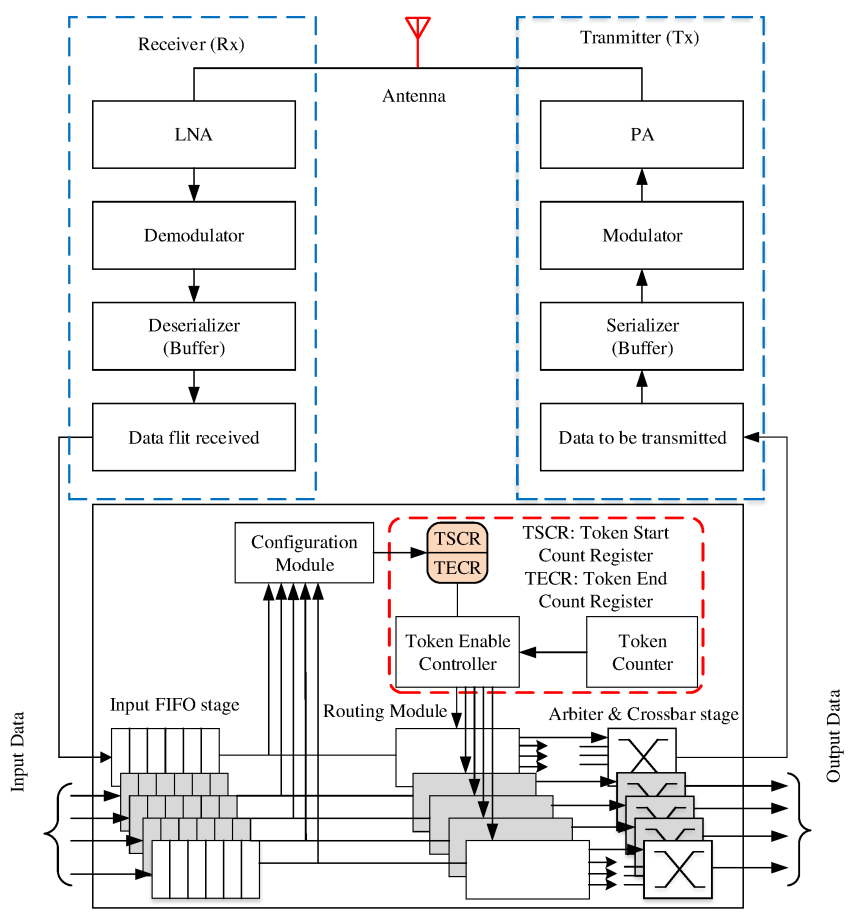

(c)

Figure 1: (a) $8 \times 8$ mesh NoC with 4 Wireless hubs in each cluster, (b) Normal router architecture and (c) Hub router architecture including the wireless transceiver portion.

destination hub using wireless transmission. Thereafter, the packet is routed to its destination. The methods to calculate the address of $\mathrm{HC}$ router are presented in Appendix A.

Broadcast Packets are communicated using WHIRL routing algorithm [29]. The structure of the head flit is modified to include a new tag called Communication Type (CT). If the CT is ' 1 ' then the packet is a broadcast packet else uni-cast packet. First, the Decision Logic checks whether the packet is a broadcast or not. If broadcast packet, it is routed to its hub connection router and all other routers in the cluster. Next, after receiving the packet, the source hub transmits the packet to all other hubs present in the WiNoC. After the broadcast packet is received by an individual hub, the packet is communicated to each router of the cluster to which the hub is associated.

\subsection{Hub Router architecture}

Figure 1(c) shows the hub router architecture. Please note that the same input port structure is present in all input ports including the wireless receiver port (WRP). Only wireless transmitter port (WTP) side Arbiter and crossbar stage has signals from 4 other input ports but all other output ports $(\mathrm{N}, \mathrm{S}, \mathrm{E}$ and W) have only one connection from WRP input port. The reason is that packets entering the hub from all ports except WRP, want to send packet using wireless communication through WTP but packet coming from wireless communication and entering from WRP input port, can go to any other output ports.

The hubs use token passing protocol to ensure fare share of the communication link usage because all of them use the same frequency. The Token counter is used to implement the token passing method which is incremented by 1 in each clock cycle and it becomes 0 after it expires to start counting again. The Token counter value is decided by the number of hubs and the duration for each hub. For example, $T_{C}=H \times T_{k}$ 
where $T_{C}$ is the Token counter range, $H$ is the number of hubs and $T_{k}$ is the duration for which the token is hold by a hub. The Token Enable Controller enables all the Routing Modules of all input ports using the Token Start Count Register (TSCR) and Token End Count Register (TECR). TSCR and TECR are configured at the start of operation by the Configuration Module. These registers will store different values in each hub denoting equal division of the total token distribution period which is same as the Token Counter range. In this way the hubs will have authority to use the wireless communication in round robin or any other manner as set by the TSCR and TECR.

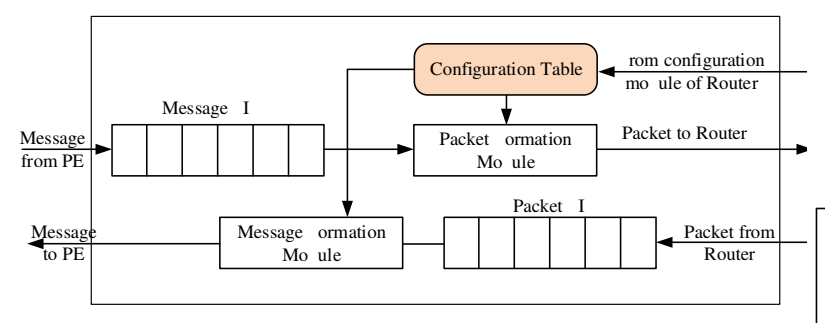

Figure 2: Network interface architecture is showr connecting a PE and the Eject port of a router.

\subsection{Network Interface (NI) architec- ture}

Figure 2 shows the Network interface (NI) architecture. The PE (processing element) sends messages to the NI where they are stored in the message FIFO. The Packet Formation Module forms packets using the Configuration table and messages from FIFO. It sends the packet to the Ejection port of the router when the port is free. The Configuration Module of the router configures the Configuration table when it receives configuration packet for this purpose. Please note that the PE does not configure anything including this table. The Message Formation Module forms messages using the Configuration Table and packets from the Eject port of the router. After that, it sends the messages to the $\mathrm{PE}$ when it is free to receive.

\subsection{Threat model}

Here we discuss the threat models considered in this work.

1. Denial of Service: In this attack, we isolate the hub(s) causing the WiNoC to behave like wired $\mathrm{NoC}$ without the benefit of wireless communication. It is also possible that the packets will wait indefinitely for a hub causing complete disruption of communication. Complete disruption of communication may happen due to congestion in normal routers because of waiting packets that want to flow through a hub.

2. Over-utilization of resources: In this attack, a resource like hub is over-utilized. This over- reliance on a resource causes congestion in the WiNoC.

3. Thermal Attacks: This is a severe form of over-utilization attack. This attack causes hyperactivity of Hub Routers causing extreme power consumption and hot spots.

4. Data stealing: In this attack, uni-cast packets are maliciously converted to broadcast packets causing leakage of sensitive data to the attacker.

\subsection{Global picture of attack scenarios}

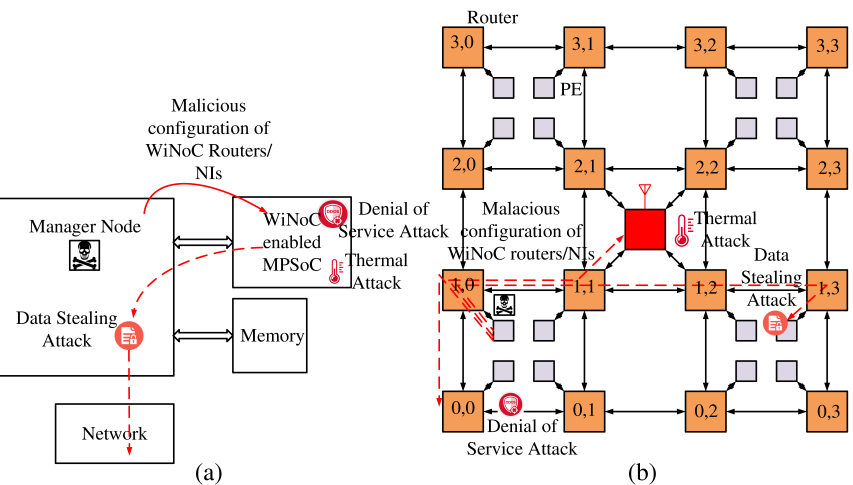

Figure 3: Two attack scenarios: (a) the host processor is compromised, and (b) an internal PE of the MPSoC is controlled by an attacker.

Here we discuss the global picture of two attack scenarios that can be used by an attacker to maliciously configure inside WiNoC to launch the attacks discussed later. Similar attack method was used in $[30,31]$ to launch attack in NoC. In the first scenario, we assume that the MPSoC using the WiNoC is connected to the outside world through a computing system (also called manager node), that is responsible for allocating and mapping applications from outside to this target system (refer Figure 3(a)). So the configuration happens as and when necessary just like a cluster computing or supercomputer system. An attacker can insert a malicious agent on this manager node first, which will then control the configuration of the WiNoC later or during runtime. In the second scenario, as shown in Figure 3(b), attacker will be allowed to run his/her applications on the MPSoC because of resource sharing on the same MPSoC. In this example, the attacker will be able to configure all the WiNoC routers from $\mathrm{PE}(1,0)$ if any security mechanism is not present. The attacker only needs to guess the address of the configuration register of each router and write the malicious data by trial and error method. Please note that the address space is not large if we consider memory mapped configuration register modification in each router of the WiNoC. Please also note that an attacker can use any one or both scenarios mentioned here to maliciously configure inside WiNoC. But depending on the configuration target, different types of attacks are possible which are discussed in more detail in next section. 


\section{New attacks against WiNoC based system with experimen- tal results}

Here we describe various new attacks namely, Malicious Threshold Configuration (MTC) Attack, Disruptive Token Passing (DTP) Attack, Data Stealing by Broadcast (DSB) Attack and Hybrid Attack against a WiNoC based system. We also provide the experimental setup and simulation results showing the effects of these attacks on the system.

\subsection{Global simulation setup}

The Wireless NoC architecture is modeled and simulated using the cycle accurate Noxim 3.0 simulator [32]. We use Snipersim 6.1 [33] for full system sim- 를 ulation of SPLASH2 [34] and PARSEC [35] bench- एँ marks to generate the traces. These traces are fed $\stackrel{\widetilde{\sigma}}{=}$ into the Noxim simulator for performance evaluation of WiNoC in terms of network latency and throughput. The Noxim simulator also reports the number of packets transmitted by the wireless hub as hub utilization. The energy consumed by the hubs depends on the hub utility and calculated in accordance with [36]. The standard Noxim simulator is used for regular WiNoC simulation. We modify the simulator to demonstrate the attack and also to show the effectiveness of our proposed countermeasures against such attacks. The system specifications are presented in Table 1. We use wormhole routing and the input buffers of the routers are 8-flit long with buffer depth of 32 bits. In order to bring out characteristics of the WiNoC architecture in the presence of both computation intensive and communication intensive workloads, we have considered four SPLASH-2 [34] benchmarks (barnes, fmm, radiosity and raytrace) and two PARSEC [35] benchmarks (blackscholes and fluidanimate). Please note that the simulation results given in this paper carry $(\mathrm{A})$ and $(\mathrm{C})$ extensions to denote under attack without countermeasure and under attack with countermeasure respectively.

\subsection{Malicious Threshold Configura- tion (MTC) Attack}

As discussed in Section 3.2.1, routers use threshold based routing method. The threshold value $T H$ is application as well as platform dependent. The mapping of applications onto an NoC platform depends on number of PEs present in the platform. Next, based on the network traffic and communication pattern, the threshold is determined. To determine the threshold values for different applications, we have conducted a set of experiments with varying threshold values. From Figure 4, we observe that different applications achieve their best (i.e., minimum) latencies for different threshold values. So a configurable threshold value will help to avoid congestion and hotspots. This value is configured in routers at the beginning of execution of the given application set. In the absence of any security measure, an at- tacker can maliciously modify this configuration value in target router(s). In this case, two types of attack scenarios may occur: (1) MTC-DOR (Denial Of Resource): The threshold value can be made very large causing abandonment of wireless hubs in the NoC i.e., denial of access of resource and (2) MTC-OU (OverUtilization): The threshold value can be made very small causing all packets to travel only through wireless hub. This causes over-utilization of the hubs and excessive power consumption.

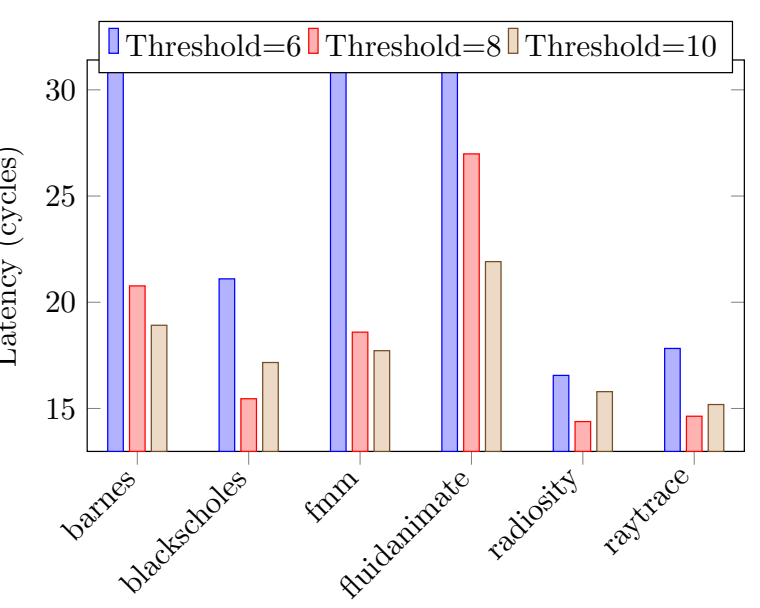

Figure 4: Latency under different threshold values for applications belonging to PARSEC and SPLASH2 benchmark suit.

\subsubsection{Simulation Setup}

We evaluate the WiNoC performance with attack (under both MTC-DOR and MTC-OU attacks) for different traffic scenarios in terms of latency, wireless hub utilization and energy. In the Noxim simulator [32], both the router and the hub design are modified to implement the proposed work. For example, our proposed threshold based routing algorithm is implemented in the Noxim simulator. In real application of the WiNoC, the threshold value is provided by the user before starting the normal operation. Currently we assume the threshold $T H$ value as 8 hops. Threshold configuration registers in normal routers (refer Figure 1(b)) are configured to 16 (greater than the maximum hop-count) and 0 to simulate MTC-DOR and MTC-OU attacks, respectively.

\subsubsection{Analysis}

Figure 5 shows run-time wireless hub utilization for both MTC-DOR and MTC-OU attacks. As shown in the figure, in MTC-DOR case, the utilization of wireless hubs is zero. Hence, energy consumed by wireless hubs are negligible (assuming all wireless hubs are in sleep mode when they are not in use to reduce the power consumption [15]). As a consequence, overall performance will be affected as wireless hubs are underutilized. Also from Figure 5, we can see that in MTC-OU case, the average increase in hub utilization across all benchmarks is 4 times compared to normal case. As a result, the average increase in 
Table 1: Simulation Setup

\begin{tabular}{|c|c|c|}
\hline Architecture & Component & Configuration \\
\hline \multirow{4}{*}{ System } & CPU & ALPHA ISA cores, out-of-order cores, 2.5GHz \\
\cline { 2 - 3 } & L1 cache & 64KB, 4-way, LRU policy, 64B line, 1-cycle latency \\
\cline { 2 - 3 } & L2 cache & 256KB, 8-way, LRU policy, 64B line, 10-cycle latency \\
\cline { 2 - 3 } & Cache coherence protocol & MESI \\
\cline { 2 - 3 } Network & Topology & 8 $\times$ 8 Mesh \\
\cline { 2 - 3 } & Routing & $\begin{array}{c}\text { Threshold based XY routing for uni-cast communication } \\
\text { WHIRL routing for broadcast communication }\end{array}$ \\
\cline { 2 - 3 } & Pipeline & 2 stages \\
\cline { 2 - 3 } & Flit size and Packet size & 32 bits and 8 flits \\
\cline { 2 - 3 } & Workload & PARSEC and Splash-2, Synthetic \\
\hline
\end{tabular}

energy consumption by wireless hubs for all applications is $137.7 \%$ as shown in Figure 6. Next, we focus our attention to Figure 7. From the figure we can observe that there is a degradation in latency under both MTC-DOR and MTC-OU attacks. In MTCDOR case, as none of the packets take the wireless route the advantage of $\mathrm{WiNoC}$ is not reflected. The increase in latency for MTC-DOR is $7.6 \%$ (on average) compared to normal case. However, in MTC-OU case, as all the packets are routed though wireless hubs, there is congestion in the network and the latency becomes very high. For this simulation setup, we consider maximum latency till 100 clock cycles.

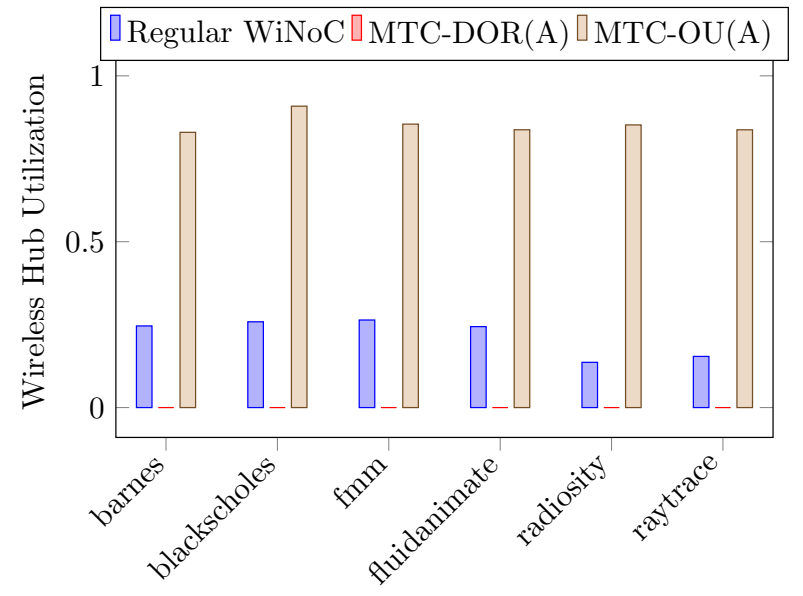

Figure 5: MTC Attack: Wireless Hub Utilizations for different benchmark applications for both MTC-DOR and MTC-OU attacks.

\subsection{Disruptive Token Passing (DTP) Attack}

In case of disruptive token passing (DTP) attack, an attacker maliciously configures TSCR and TECR in target hub(s) (refer Figure 1(c)). There are mainly 2 types of DTP attack: 1)DTP-DOS (Denial Of Service): the attacker can make the duration 0 causing denial of service of a particular hub and 2)DTPAHT (All Hubs holding Token): The duration can be made to the whole counter range for all hubs. This will make all the hubs holding the token all the time causing collisions and packet drops between different hub communications. This also increases the power consumption of the hubs.

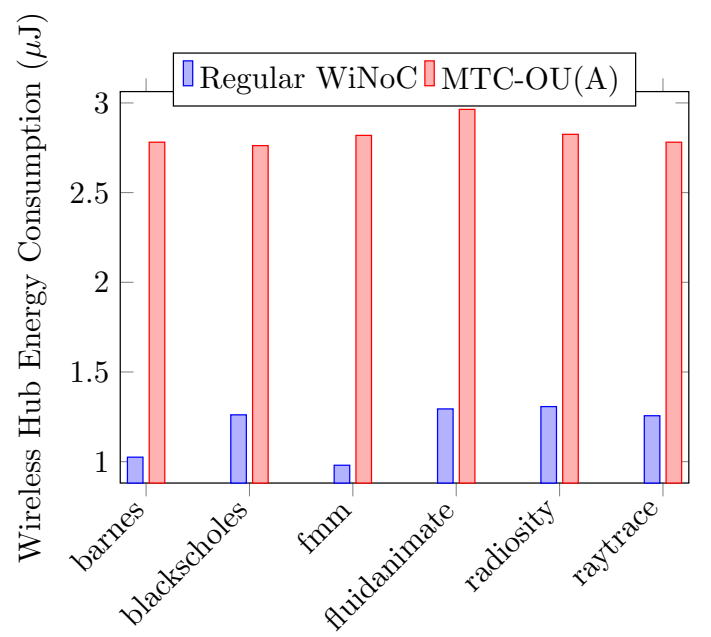

Figure 6: MTC Attack: Wireless Hub Energy Consumption for different benchmark applications for MTC-OU attack.

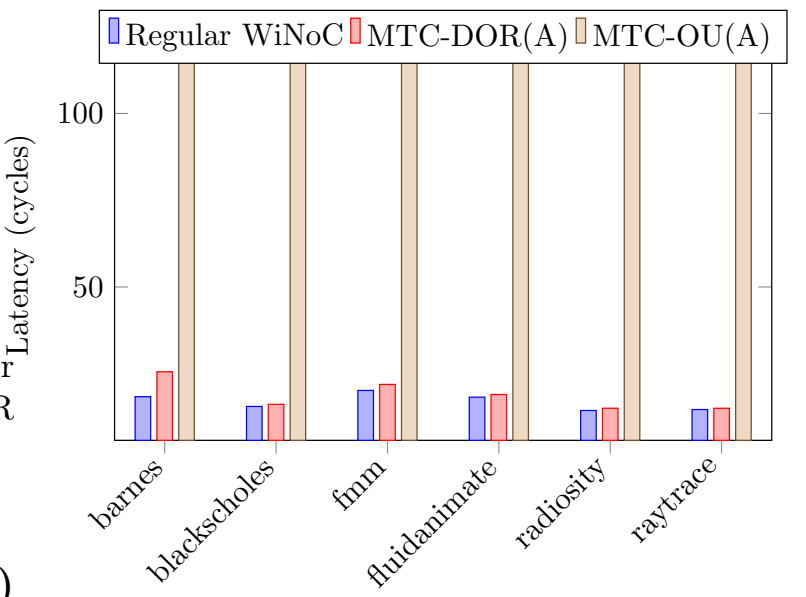

Figure 7: MTC Attack: Average Network Latency for different benchmark applications for both MTC-DOR and MTC-OU attacks.

\subsubsection{Simulation Setup}

We present the performance of WiNoC under both DTP-DOS and DTP-AHT attacks under the aforementioned traffic scenarios in terms of throughput, packet loss and wireless hub utilization. The token passing mechanism of Noxim simulator [32] is modified to simulate both DTP-DOS and DTP-AHT attacks. In particular, the contents of Token Start 
Count Register (TSCR) and Token End Count Register (TECR) are modified to implement the DTP attacks.

\subsubsection{Analysis}

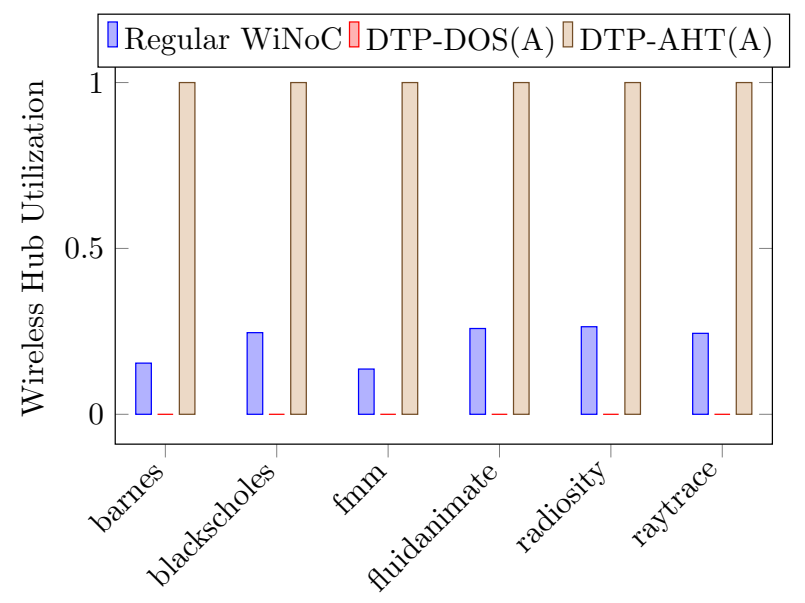

Figure 8: DTP Attack: Wireless Hub Utilizations for different benchmark applications for both DTP-DOS and DTP-AHT attacks.

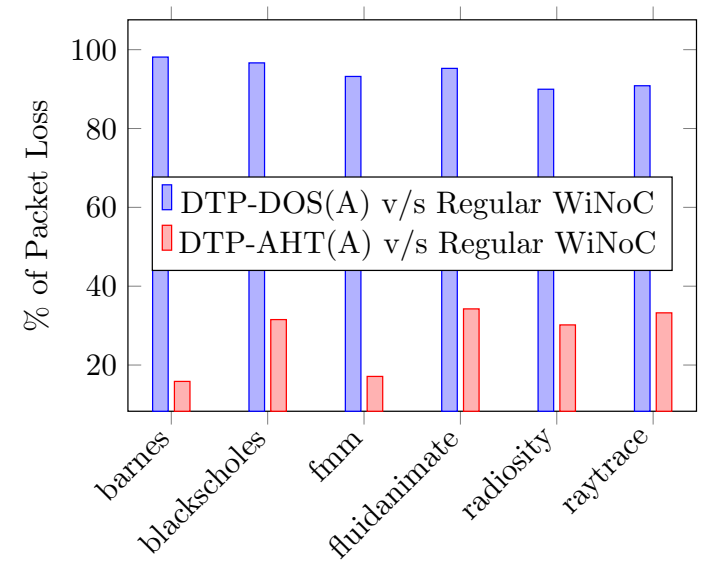

Figure 9: DTP Attack: Packet Loss for different benchmark applications for both DTP-DOS and DTP-AHT attacks.

The Wireless Hub Utilization for DTP attack is depicted in Figure 8. For the DTP-DOS attack, the hub utilization is negligible as none of the hubs have the token and packet transmission from hubs are stopped. However, it can be observed that the hub utilization is $100 \%$ for DTP-AHT attack. As all hubs have the token, they always remain active irrespective of packet transmission. This results in high energy consumption by the hubs. In our experiment, we found that the maximum hub energy to be $32.3 \mu \mathrm{J}$. In case of DTP-DOS, packets are directed towards the hub for wireless transmission. As no hub has token, these packets wait in their path causing congestion in the network. This results in packet loss which is the number of packets that could not be injected into the system due to unavailability of buffer space. The average packet loss for all the application is $94 \%$ in case of DTP-DOS attack. This is depicted in Figure 9.

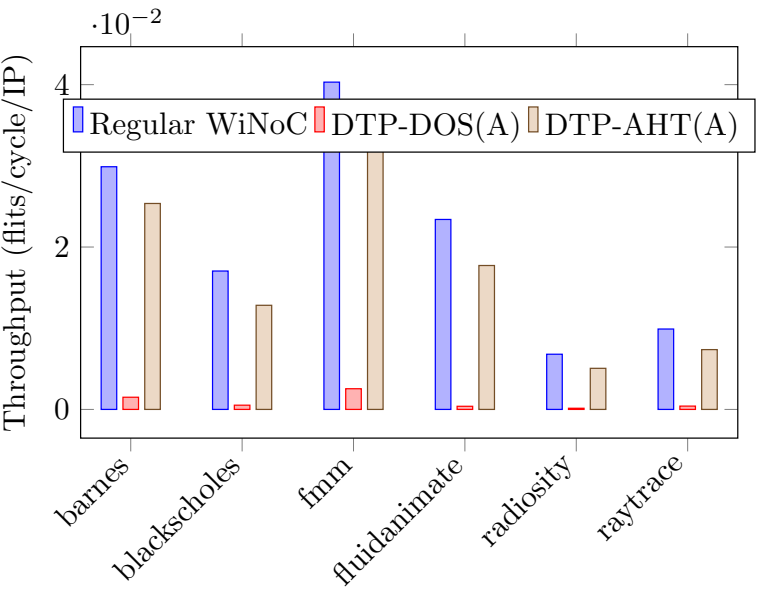

Figure 10: DTP Attack: Average Network Throughput for different benchmark applications for both DTP-DOS and DTP-AHT attacks.

Also, from the figure we observe that there is packet loss in case of DTP-AHT. The hubs operate in a half duplex mode i.e., a hub cannot transmit and receive at the same time. If all of them have the token, then all are configured as transmitter. Thus, packets will be transmitted from the source hub but will not be received at the destination hub. The average percentage of packet loss is $27 \%$ for DTP-AHT compared to regular WiNoC. The packet loss during both attacks also cause average network throughput drop (more for DTP-DOS than DTP-AHT) compared to regular WiNoC. The drop in throughput for all applications, on average is $23.1 \mathrm{x}$ and $1.23 \mathrm{x}$ for DTP-DOS and DTPAHT, respectively. This is depicted in Figure 10.

\subsection{Data Stealing by Broadcast (DSB) Attack}

In case of secure communication inside the WiNoC, the wireless communication is not used because all the hubs along with the intended receiver hub can snoop into the packets. This special communication is only possible because the packets carry a type bit that is recognized by all routers. The packets are formed inside network interface of the sender PE using a configuration table (refer Figure 2) which translates the messages received from the PE to a set of packets. This table is also configured at the beginning of WiNoC operation and can be maliciously modified by an attacker. The attacker can modify the packet type from special to normal and from uni-cast to broadcast. In case of DSB attack, the packets will be transmitted to all the routers instead of a particular destination router.

Note that, the term PE also denotes the memory controller that connects the external memory with the WiNoC fabric. In a shared memory system, the PE (processing element) that is responsible for encryption or decryption for secure communication, loads the secret key from external memory. Even if the PE uses local memory to store the key and performs security operations, it needs to load from memory in a regular basis because in a practical system the keys 
are not fixed for a long time. The attacker can maliciously modify the Configuration Table inside the NI of the router connecting the memory controller. In this way, the attacker will obtain the packets whenever the secure PE loads the key. Please note that this attack is possible because it does not depend on any software vulnerability or OS vulnerability but uses a network level attack. Also note that the DSB attack can target any sensitive data (for example, any personal data) and not only secret key.

\subsubsection{Simulation Setup}

For experimentation, we assume that 4 Memory Controllers (MCs) are present in the WiNoC at the 4 corners (refer Figure $1(\mathrm{a})$ ) i.e., $P E_{0,0}, P E_{0,7}, P E_{7,0}$ and $P E_{7,7}$. We assume that a secret key is pre-loaded securely to the memory connected to $P E_{0,0}$. This secret key is needed to decrypt the encrypted data available at the memory connected to $P E_{7,0}$. In case of attack, all the uni-cast packets injected into the WiNoC from $P E_{0,0}$ are converted to broadcast by changing the CT tag in packets from ' 0 ' to ' 1 '.

\subsubsection{Analysis}

Figure 11 shows router utility factor (RUF) for all the routers present in the network. During normal operation (i.e., without any attack), the $P E_{7,4}$ initiates a request and sends it to the $P E_{0,0} . \quad P E_{0,0}$ processes the request and sends the requested key to the initiator $\mathrm{PE}$. On receiving the key, the $P E_{7,4}$ uses it to retrieve information from the $P E_{7,0}$. This is depicted in Figures 11(a) and 11(b), respectively. Next, we launch an attack on the NI connecting the $P E_{0,0}$ and router $_{0,0}$, and the packet which was meant for $P E_{7,4}$ is converted into a broadcast packet. So, the packet is broadcasted to all the PEs present in the network. Now, every PE has the key and using this key they can access the data through $P E_{7,0}$. In this example, $P E_{2,2}$ is a malicious $P E$. Thus, after receiving the key, $P E_{2,2}$ retrieves the encrypted data through $P E_{7,0}$. This is depicted in Figures 11(c) and 11(d) respectively. Figure 11(d) also shows the data transmission between $P E_{7,4}$ and $P E_{7,0}$ apart from the data stealing by $P E_{2,2}$.

\subsection{Hybrid Attack}

An attacker combines different attacks to launch a hybrid attack. For example, the attacker can combine MTC-OU attack where all communications become wireless and DTP-AHT attack when all hubs are active all the time. Worst case situation results in a thermal attack, where the attacker combines DSB (targeting all or a group of routers' network interfaces) with MTC-OU and DTP-AHT. In that case, all the hubs are sending to all other hubs increasing the packet flow and power consumption in the whole chip.

\subsubsection{Simulation Setup}

We use the PARSEC benchmark application "fluidaminate" to evaluate the thermal attack on the
WiNoC shown in Figure 1(a). For this purpose, we configure the normal routers for MTC-OU attack, hub routers for DTP-AHT attack and NIs for DSB attack. The power consumption of the routers, the hubs, and the PEs are estimated and are fed to the Hotspot tool [37] to calculate the temperature of the chip. The hub power is considered to be similar to [36] and depends on the hub utility. Similarly, we calculate RUFs for all the routers present in the $\mathrm{WiNoC}$. These values are fed to the Orion tool [38] to calculate individual router power. The average power consumption of a $\mathrm{PE}$ is extracted from Snipersim [33]. In addition to the power of the individual components, Hotspot also requires floor-plan of the chip. In this paper, we consider a chip of $20 \mathrm{~mm} \times 20 \mathrm{~mm}$, consisting of 64 cores arranged in $8 \times 8$ mesh structure. We use the grid model of HotSpot with a grid size of $1024 \times 1024$.

\subsubsection{Analysis}

The temperature profiles of the chip during normal operation and thermal attack are given in Figures 12 (a) and 12(b) respectively. During normal operation, we observe a relatively uniform temperature profile for the entire chip. This is because the utility of both the routers and hubs are quite low for the "fluidaminate" application specific traffic. The zoomed portion in Figure 12(a) shows tiles associated with a hub with $2 \%$ utility factor. Next, during thermal attack, the overall chip temperature is high, with the tiles associated with wireless hubs exhibiting the highest temperature. Due to the combination of MTC-OU and DTP-AHT attacks, the overall energy consumption of wireless hubs are significantly higher compared to regular WiNoC. Also, DSB attack forces uni-cast packets to broadcast. Thus, the number of packets processed by the routers increases, which in turn increases the router power consumption. This rise in temperature profile of the chip may degrade the system performance and in extreme cases leads to system failure.

\section{Countermeasures and com- parison with related works}

In this Section, we discuss various countermeasures against the attacks considered in this paper. We also discuss the comparison with related works.

\subsection{Source Destination (SD) check- ing mechanism: countermeasure against MTC Attack}

We implement the Source Destination (SD) checking mechanism as given in Algorithm 2 in Decision logic (refer Figure 1(b)) before performing the threshold based routing in RLU. SD mechanism is used to conditionally bypass the threshold based method and directly use the Routing logic unit for XY routing.

Algorithm 2 compares the manhattan distance between the source and destination routers and their corresponding hub-connection routers with distance 


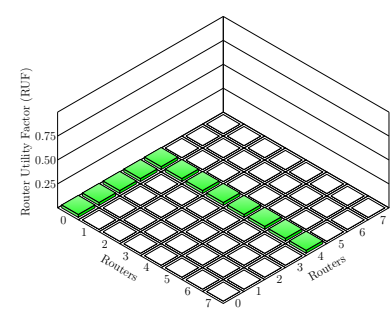

(a)

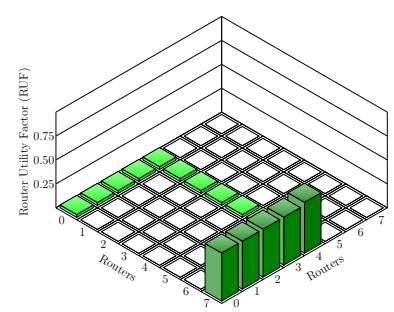

(b)

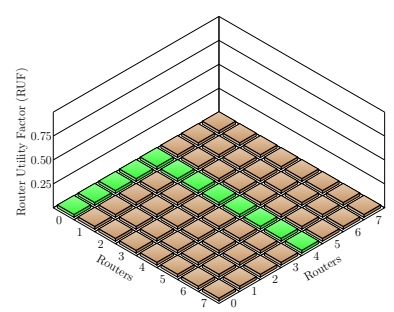

(c)

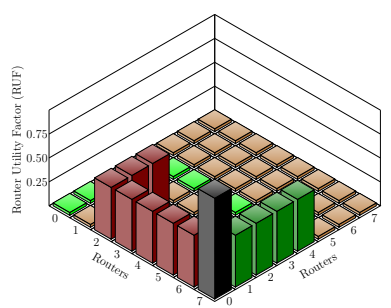

(d)

Figure 11: Normal execution case : (a) key transfer and (b) data transaction. Under attack case: (c) key transfer and (d) data transaction.

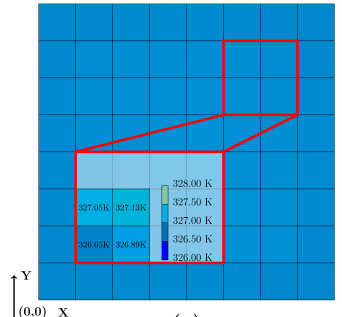

(a)

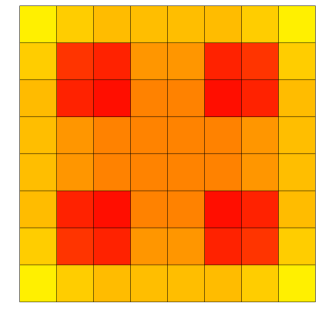

(b)

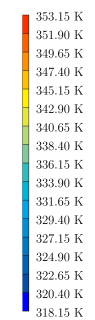

Figure 12: Temperature profile of the chip under (a) normal operation and (b) under thermal attack.

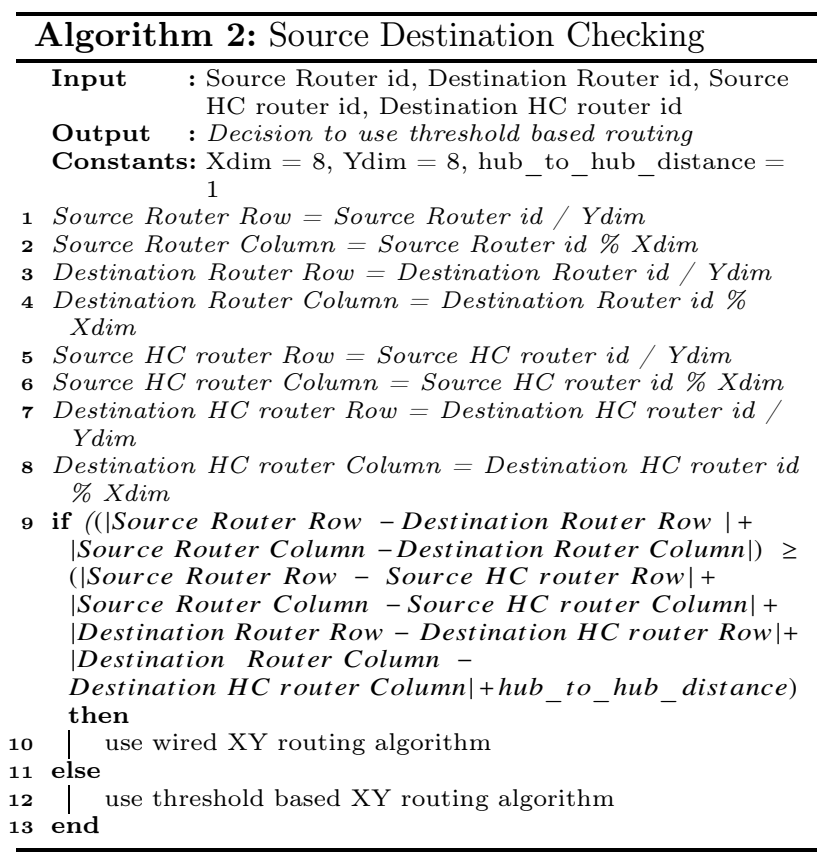

between source and destination routers. If the later is greater, then the packet is transmitted using wired communication. However, if the former is greater then it is compared with the threshold $T H$. This method helps to stop the flow of all packets towards the hubs and helps to counter the MTC-OU attack.

\subsubsection{Simulation Setup}

To implement the countermeasure for MTC attack, we have modified the routing algorithm present in the Noxim simulator. We use Algorithm 2 before evoking Algorithm 1 for routing packets from the source to destination node. As defined in Section 3.2.1, the source and destination addresses are obtained from the head flit of the packet. Next, Algorithms 3 and 4 from Appendix A are invoked to calculate the source and destination cluster ids and sub-cluster ids. This data is used to calculate the position of $\mathrm{HC}$ routers for source and destination nodes using Algorithm 5 given in Appendix A. These data are given as input to Algorithm 2, which determines the use of wireless hub for packet transmission.

\subsubsection{Analysis}

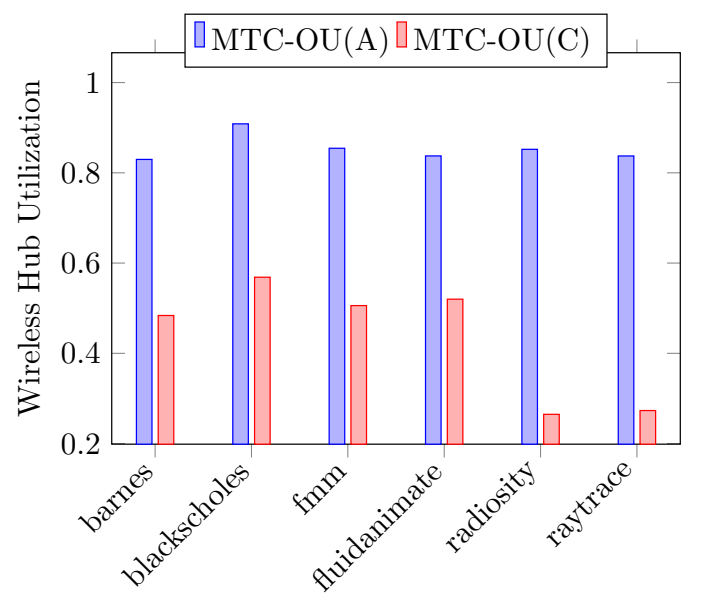

Figure 13: MTC Attack: Wireless Hub Utilizations for different benchmark applications without and with countermeasure under MTC-OU attack.

The source destination checking mechanism helps to reduce the wireless hub utilization caused by MTCOU attack as shown in Figure 13. The figure shows a $49 \%$ decrease (on average) in wireless hub utilization under MTC-OU attack when countermeasure is present. The energy consumption by the hubs also decreases (on average 38\%) due to the decrease in wireless hub utilization in the presence of proposed countermeasure. This is depicted in Figure 14. During normal operation, the threshold is set to 8 hops. In case of MTC-OU attack, this value is changed to zero causing all the packets to flow through the wireless route. Using our SD countermeasure technique, we restrict the packet flow using the condition present in Algorithm 2. Therefore, there is an obvious improvement in average network latency in presence of proposed countermeasure under MTC-OU attack case as shown in Figure 15. 


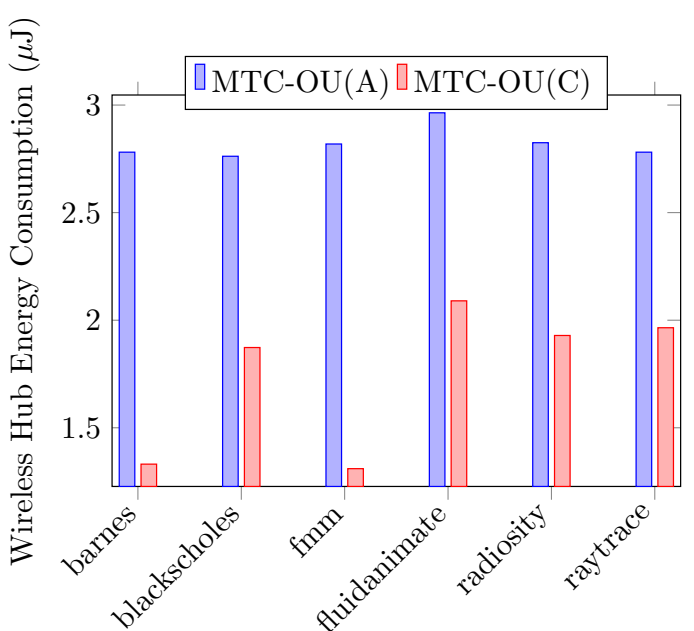

Figure 14: MTC Attack: Wireless Hub Energy Consumption for different benchmark applications without and with countermeasure under MTC-OU attack.

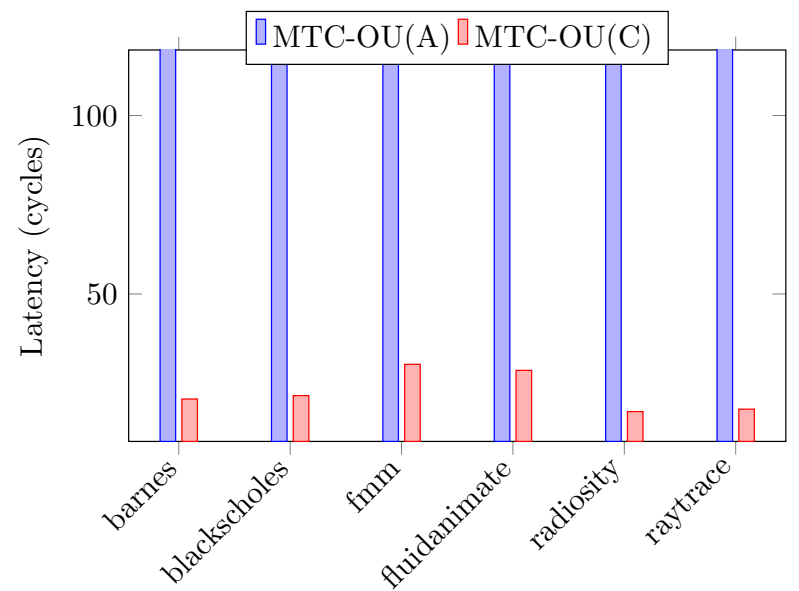

Figure 15: MTC Attack: Average Network Latency for different benchmark applications without and with countermeasure under MTC-OU attack.

\subsection{Detour mechanism: countermea- sure against DTP Attack}

We propose a new wireless hub access mechanism in the WiNoC. The DTP-DOS attack is detected by introducing a token wait counter (TWC) in each hub. TWC is incremented by 1 at each clock cycle if the token is not enabled. When the token is enabled in the hub, TWC is reset. TWC starts counting again when the token is disabled. To detect DTP-DOS attack, we check the most significant bit (MSB) of the TWC. If the MSB is 1 then the hub is under DTPDOS attack because TWC counted for many clock cycles without the token. To detect the DTP-AHT attack, we have introduced two counters in the transmitter and receiver sections of the hub. These are Packet Transmission Counter (PTC) and Packet Reception Counter (PRC). Whenever a hub transmits a flit the PTC is incremented by one, on the other hand whenever a hub receives a flit the PRC is incremented by one. Also, after a receiver hub receives the tail flit, it sends an acknowledgment message to the transmitter hub. This is a self generated single flit packet.
We keep two checks for detection of DTP-AHT attack. First, we check if the $4^{t h}$ bit of PTC is ' 1 ' or ' 0 '. We select the $4^{\text {th }}$ bit of PTC because 2 packets (16 flits) have been considered as threshold. This can be modified depending on the requirement of the user. Next, if the $4^{\text {th }}$ bit is ' 1 ', we check the PRC is all zero or not. If PRC is all zero, then the hub is under DTP-AHT attack. In the above mentioned mechanisms, if an attack is detected in the hub, the hub is switched off. A signal is sent to the hub connection routers in the local cluster (the cluster in which the hub belongs) informing the unavailability of the hub for packet transmission. Packets in the hub connection router awaiting for wireless transmission through the hub are re-directed towards the destination node using wired NoC. This process is implemented by using a detour based routing mechanism implemented in the Routing Logic Unit in the HC routers. The flowchart for this detour based routing is shown in Figure 16. Please note that 'Dest dst' denotes the manhattan distance between the source and destination routers.

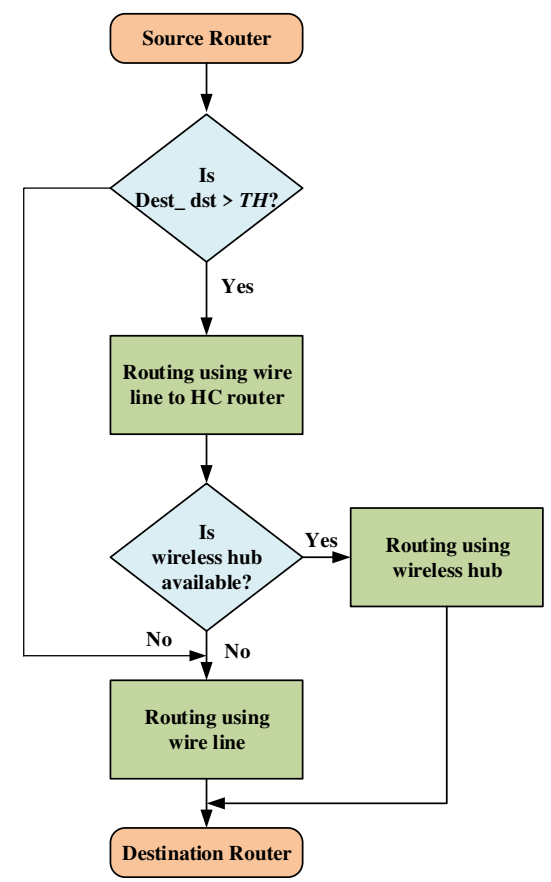

Figure 16: Flowchart of detour based routing mechanism.

\subsubsection{Simulation Setup}

We have modified the hub architecture in Noxim simulator to detect the attacks and implement the countermeasures for DTP-DOS and DTP-AHT attacks. We have added TWC in hubs to get the wait time before its token is enabled. In transmitter and receiver sections of the hub, two counters (PTC and PRC) have been implemented to count the number of transmitted and received flits. A new signal which indicates the availability of the hub is introduced between the hub and the respective hub connection routers. Also, the detour based routing algorithm for packet transmission in case of attack is incorporated in the 
Noxim

\subsubsection{Analysis}

Our proposed countermeasures against DTP attacks help to reduce the packet loss. Thus, in presence of proposed countermeasures, the throughput of the system is improved by $1.21 \mathrm{x}$ and $23 \mathrm{x}$ under DTPAHT (refer Figure 17(a)) and DTP-DOS (refer Figure 17(b)) attacks respectively. Next, we focus our attention to the average network latency. As the hubs are switched off, all packets are communicated by wired routes. In this situation, the advantages of wireless NoC compared to wired NoC cease to exist. Thus there is a performance degradation under DTP attacks in presence of proposed countermeasures compared to regular WiNoC without any attack, as shown in Figure 18. The figure shows that the average latency increase for all the benchmarks with our countermeasures under DTP-DOS and DTP-AHT attacks are $14.65 \%$ and $14.23 \%$ respectively, compared to regular WiNoC.

\subsection{Countermeasures against DSB and hybrid Attacks}

If an attacker launches a hybrid attack combining DTP-DOS and MTC-OU attacks, we need to combine both countermeasures (Source destination checking and detour mechanism). Figure 19 shows the average network latency for different benchmark applications under hybrid attack with combined countermeasure in comparison with individual countermeasures. The combined countermeasure shows $9.46 \%$ and $24.55 \%$ degradation in latency compared to the countermeasure against MTC-OU and DTP-DOS attacks respectively. This is because countermeasure against MTCOU restricts the flow checking mechanism defined by Algorithm 2. However, the number of packets flowing toward the wireless hub increases as the predefined threshold is altered to zero by the attacker. Next, a bypass routing mechanism presented in Figure 16 is used to route the packets to their destination using the wired NoC. This is because as a countermeasure against DTP-DOS, the wireless hubs are switched-off. Hence, the latency increases when two countermeasures are implemented together. But note that the extra overhead is comparatively small if we consider the protection against the hybrid attack.

All the attacks considered in this paper are mainly launched by taking advantage of insecure router configuration method. There are many configurable portions in a WiNoC based system. As a countermeasure, we need to ensure that only trusted configuration source is allowed to configure both types of routers (normal and hub) and various parts of those routers. Existing source authentication methods like [39] can help to implement lightweight countermeasures in a security critical WiNoC system. In this existing solution, the authenticity of the configuration source is first checked before it is allowed to configure a destination. Various watermarking techniques, a stream authentication method and combinations of these methods are used to transfer source authentication information to destination where it is checked before starting the configuration.

In addition to that, we can ensure that a secure core only accesses a fixed memory controller which has pre-shared keys with it and it uses existing cryptographic algorithms like AES to encrypt the packet contents before sending it to the secure core. In this way, only the secure core can decrypt the contents and an attacker cannot get the content even if he/she got the packets in some manner. Please note that here, we do not assume that all the PEs or all the memory controllers use encryption to hide contents of the packets. This is necessary to limit the overhead associated with symmetric cipher like AES impacting total performance of the system when most portion of it may not require such level of security.

Even with cryptographic solutions, an attacker can try to access the memory by requesting read or write requests to the memory controller. Even if the attacker does not know the secret key, he/she can modify the memory contents with garbage values by performing write operation. As a countermeasure we need a memory access control method that can ensure that an attacker cannot access the memory to perform illegal tasks. Authors in [40] have used Role Based Access Control (RBAC) model to implement memory access control and a WiNoC based system can benefit from this solution. In this solution, every packet has a role id and every role has distinct permission to access a memory location and/or perform some task. Here an attacker cannot access a memory without obtaining the correct role through a secure authentication method.

\subsection{Comparison with related works}

Authors in [2] have considered DOS attack by intentional violation of collision prevention measures by attacker, Spoofing attack by giving false identity of attacker as legitimate source and Eavesdropping attack during broadcast operation by a source. They propose to use stream cipher called Py to protect from eavesdropping attack and it causes 5 cycles of latency overhead ( $46 \%$ increase over the unencrypted delay at $64 \mathrm{Gbps})$. In our case, we only consider specialized attacks which are specific to a WiNoC based system. The attack methods given in [2] are not realistic because the Hardware Trojan that is assumed to cause all the attacks can also modify the behavior of their proposed countermeasures. In our case, we provide much more realistic attack methods and also show results using various benchmark applications to show their effects. We also provide the countermeasures that are resilient to these attacks.

Authors in [3] have also considered spoofing, replay, and message modification attacks. Replay attack happens by repeating valid packets which causes false operation and modification attack happens by altering the content of messages sent by legitimate senders. They assume that there is a fraudulent device within the wireless coverage area which is tuned at the target wireless frequency spectrum. This de- 


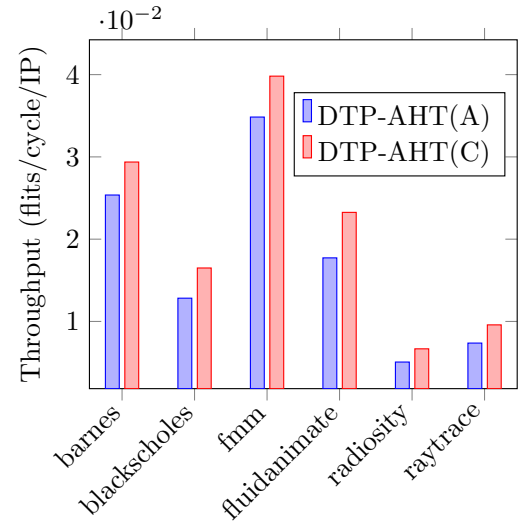

(a)

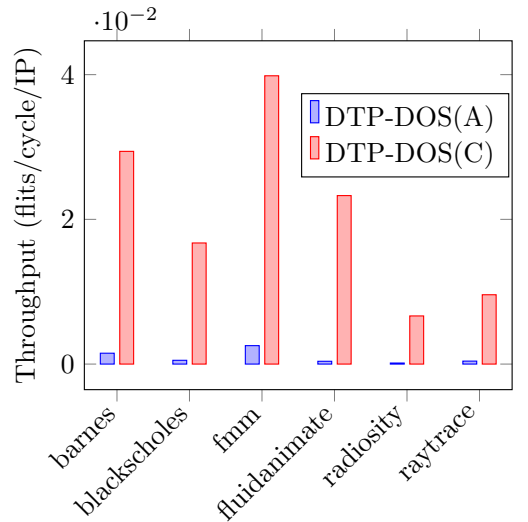

(b)

Figure 17: DTP Attack: Average Network Throughput for different benchmark applications under (a) DTPAHT and (b) DTP-DOS attacks for both without and with countermeasures.

Table 2: Summary of comparison with related works

\begin{tabular}{|c|c|c|c|c|}
\hline & {$[2]$} & {$[4]$} & {$[3]$} & Our work \\
\hline Attack types & $\begin{array}{c}\text { DOS, Spoofing, } \\
\text { Eavesdropping }\end{array}$ & DOS & $\begin{array}{c}\text { Spoofing, Replay, } \\
\text { Message modification }\end{array}$ & $\begin{array}{c}\text { DOS (MTC-DOR, } \\
\text { DTP-DOS, DTP-AHT), } \\
\text { Overutilization (MTC-OU), } \\
\text { Data stealing (DSB) }\end{array}$ \\
\hline Attack method & Hardware Trojan & Hardware Trojan & $\begin{array}{c}\text { Fradulent device placed } \\
\text { within wireless } \\
\text { coverage area }\end{array}$ & Malicious configuration \\
\hline $\begin{array}{c}\text { Performance overhead } \\
\text { due to } \\
\begin{array}{c}\text { countermeasure during } \\
\text { normal operation }\end{array}\end{array}$ & $46 \%$ at 64 Gbps & $\begin{array}{c}\text { Depends on the } \\
\text { derived topology } \\
\text { which is depended } \\
\text { on application }\end{array}$ & More than 30\% & No degradation \\
\hline
\end{tabular}

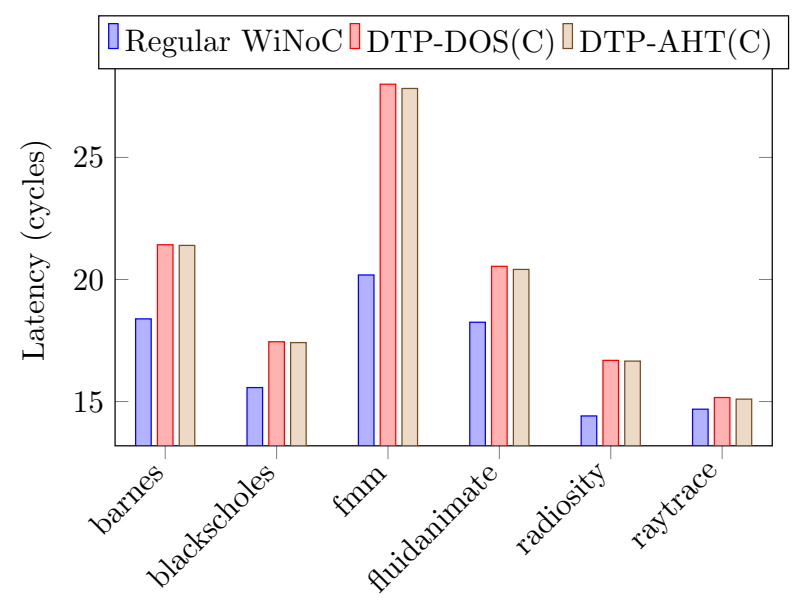

Figure 18: DTP Attack: Average Network Latency for different benchmark applications for regular WiNoC without any attack and also under DTP-DOS and DTP-AHT attacks in presence of proposed countermeasures.

vice interferes the communication in the $\mathrm{WiNoC}$ to launch attacks. They have proposed a hash based countermeasure to protect from the attacks. Please note that this solution is applied to all the packets and causes more than $30 \%$ performance overhead compared to the system without any security measures. We also use a countermeasure for source authentication [39] but that is used before starting normal oper-

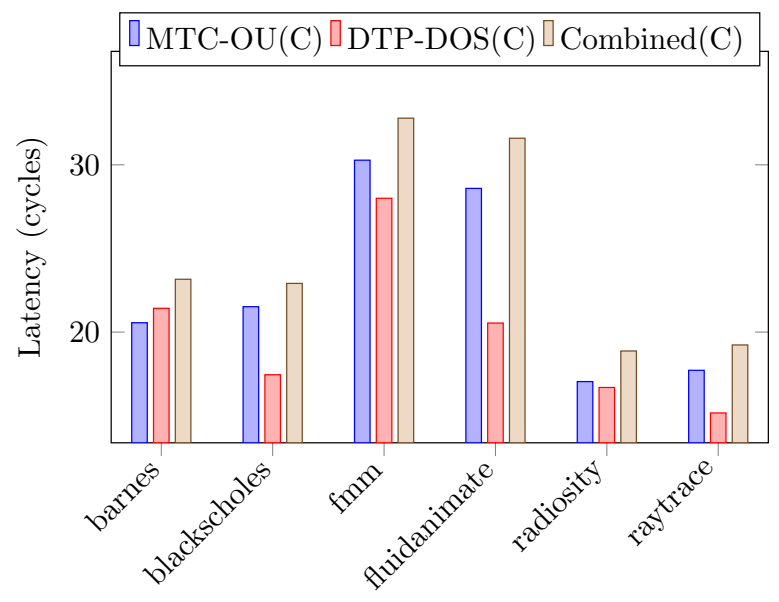

Figure 19: Hybrid Attack (MTC-OU and DTP-DOS): Average Network Latency for different benchmark applications under hybrid attack with combined countermeasure in comparison with individual countermeasures.

ation of the WiNoC and hence does not cause any performance degradation. Our additional countermeasures of memory access control [40] do not cause any performance degradation and only the encryption based countermeasure mentioned in Section 5.3 will cause performance issues but it is limited only to the secure core communicating with a specific memory controller. 
Authors in [4] have considered DOS attack due to Hardware Trojan. They have not proposed any countermeasure but have proposed to minimize the effect of attack to some extent by using a new network topology which is dependent on the application running in the system. That means the WiNoC cannot run any other application other than the one considered during design. Otherwise the DOS attack resistance will not be effective. Our countermeasures are not application dependent and this allows the WiNoC to facilitate parallel applications to run in the system.

A summary of comparison with related works is given in Table 2 .

\section{Conclusions}

In this paper, we have described Malicious Threshold Configuration (MTC) Attack, Disruptive Token Passing (DTP) Attack, Data Stealing by Broadcast (DSB) Attack and Hybrid Attack against the WiNoC based system. We have also provided the experimental setup and simulation results showing the effects of these attacks on the system. We have shown that MTC-DOR attack causes wireless hub utilization to be 0 and MTC-OU attack increases it around 4 times compared to regular WiNoC. Also MTC-OU attack increases average network latency by many orders of magnitude compared to regular WiNoC causing overall system performance degradation. We have also shown that the wireless hub utilization becomes 0 for DTP-DOS attack and around $100 \%$ for DTP-AHT attack. The network throughput drops around 23.1x and $1.23 \mathrm{x}$ under DTP-DOS and DTP-AHT attacks respectively. In addition, we have provided countermeasures that can protect from these attacks without causing considerable performance overhead of the system. Our proposed countermeasure against MTC-OU attack i.e., Source Destination checking mechanism decreases wireless hub utilization by $49 \%$ and network latency by many orders of magnitude compared to without countermeasure, causing system performance improvement. Our proposed countermeasure against DTP attacks i.e., detour mechanism improves the network throughout by $1.21 \mathrm{x}$ and $23 \mathrm{x}$ under DTPAHT and DTP-DOS attacks respectively compared to without countermeasure cases.

In future, we would like to consider hardware trojans which were out of scope of this paper. We will also consider FDMA and CDMA techniques for attacks and their countermeasures in our future work.

\section{References}

[1] S. Evain and J. Diguet, "From noc security analysis to design solutions," in IEEE Workshop on Signal Processing Systems Design 85 Implementation, ser. VLSISP, Nov 2005.

[2] B. Lebiednik, S. Abadal, H. Kwon, and T. Krishna, "Architecting a secure wireless network-on-chip," in 2018 Twelfth IEEE/ACM International Symposium on Networks-on-Chip (NOCS), 2018.

[3] F. Pereñíguez García and J. L. Abellán, "Secure communications in wireless network-on-chips," in Pro- ceedings of the 2Nd International Workshop on Advanced Interconnect Solutions and Technologies for Emerging Computing Systems, ser. AISTECS '17, 2017.

[4] A. Ganguly, M. Y. Ahmed, and A. Vidapalapati, "A denial-of-service resilient wireless noc architecture," in Proceedings of the Great Lakes Symposium on VLSI, ser. GLSVLSI '12, 2012.

[5] P. P. Pande, A. Ganguly, K. Chang, and C. Teuscher, "Hybrid wireless network on chip: A new paradigm in multi-core design," in 2009 2nd International Workshop on Network on Chip Architectures, Dec 2009, pp. $71-76$.

[6] C. Wang, W. Hu, and N. Bagherzadeh, "A wireless network-on-chip design for multicore platforms," in 2011 19th International Euromicro Conference on Parallel, Distributed and Network-Based Processing, 2011.

[7] S.-B. Lee, S.-W. Tam, I. Pefkianakis, S. Lu, M. F. Chang, C. Guo, G. Reinman, C. Peng, M. Naik, L. Zhang, and J. Cong, "A scalable micro wireless interconnect structure for cmps," in Proceedings of the 15th Annual International Conference on Mobile Computing and Networking, ser. MobiCom '09, 2009.

[8] S. Deb, K. Chang, X. Yu, S. P. Sah, M. Cosic, A. Ganguly, P. P. Pande, B. Belzer, and D. Heo, "Design of an energy-efficient cmos-compatible noc architecture with millimeter-wave wireless interconnects," IEEE Transactions on Computers, vol. 62, no. $12,2013$.

[9] A. Ganguly, K. Chang, S. Deb, P. P. Pande, B. Belzer, and C. Teuscher, "Scalable hybrid wireless network-on-chip architectures for multicore systems," IEEE Transactions on Computers, vol. 60, no. 10, 2011.

[10] A. Ganguly, P. Wettin, K. Chang, and P. Pande, "Complex network inspired fault-tolerant noc architectures with wireless links," in Proceedings of the Fifth ACM/IEEE International Symposium, 2011.

[11] D. DiTomaso, A. Kodi, S. Kaya, and D. Matolak, "iwise: Inter-router wireless scalable express channels for network-on-chips (nocs) architecture," in 2011 IEEE 19th Annual Symposium on High Performance Interconnects, 2011.

[12] M. Alaei and F. Yazdanpanah, "H2wnoc: A honeycomb hardware-efficient wireless network-on-chip architecture," Nano Communication Networks, vol. 19, pp. 119 - 133, 2019.

[13] A. Vidapalapati, V. Vijayakumaran, A. Ganguly, and A. Kwasinski, "Noc architectures with adaptive code division multiple access based wireless links," in 2012 IEEE International Symposium on Circuits and Systems (ISCAS), May 2012, pp. 636-639.

[14] R. Wu, Y. Wang, and D. Zhao, "A low-cost deadlock-free design of minimal-table rerouted xyrouting for irregular wireless nocs," in 2010 Fourth ACM/IEEE International Symposium on Networkson-Chip, 2010.

[15] H. K. Mondal, S. H. Gade, S. Kaushik, and S. Deb, "Adaptive multi-voltage scaling with utilization prediction for energy-efficient wireless noc," IEEE Transactions on Sustainable Computing, vol. 2, no. 4, 2017. 
[16] A. Asaduzzaman, K. K. Chidella, and D. Vardha, "An energy-efficient directory based multicore architecture with wireless routers to minimize the communication latency," IEEE Transactions on Parallel and Distributed Systems, vol. 28, no. 2, pp. 374-385, Feb 2017.

[17] D. Zhao, Y. Wang, J. Li, and T. Kikkawa, "Design of multi-channel wireless noc to improve onchip communication capacity!" in Proceedings of the Fifth ACM/IEEE International Symposium, May 2011, pp. 177-184.

[18] I. E. Masri, T. Le Gouguec, P. Martin, R. Allanic, and C. Quendo, "Integrated dipole antennas and propagation channel on silicon in ka band for winoc applications," in 2018 IEEE 22nd Workshop on Signal and Power Integrity (SPI), May 2018, pp. 1-4.

[19] M. O. Agyeman, Q. Vien, and T. Mak, "An analytical channel model for emerging wireless networkson-chip," in 2016 IEEE Intl Conference on Computational Science and Engineering (CSE) and IEEE Intl Conference on Embedded and Ubiquitous Computing (EUC) and 15th Intl Symposium on Distributed Computing and Applications for Business Engineering (DCABES), Aug 2016, pp. 9-15.

[20] J. O. Sosa, O. Sentieys, and C. Roland, "A diversity scheme to enhance the reliability of wireless noc in multipath channel environment," in 2018 Twelfth IEEE/ACM International Symposium on Networkson-Chip (NOCS), Oct 2018, pp. 1-8.

[21] D. W. Matolak, S. Kaya, and A. Kodi, "Channel modeling for wireless networks-on-chips," IEEE Communications Magazine, vol. 51, no. 6, pp. 180-186, June 2013.

[22] X. Yu, S. P. Sah, S. Deb, P. P. Pande, B. Belzer, and D. Heo, "A wideband body-enabled millimeterwave transceiver for wireless network-on-chip," in 2011 IEEE 54th International Midwest Symposium on Circuits and Systems (MWSCAS), Aug 2011, pp. $1-4$.

[23] S. H. Gade, S. Garg, and S. Deb, "Ofdm based high data rate, fading resilient transceiver for wireless networks-on-chip," in 2017 IEEE Computer Society Annual Symposium on VLSI (ISVLSI), July 2017, pp. $483-488$.

[24] J. Ortiz Sosa, O. Sentieys, and C. Roland, "Adaptive transceiver for wireless noc to enhance multicast/unicast communication scenarios," in 2019 IEEE Computer Society Annual Symposium on VLSI (ISVLSI), July 2019, pp. 592-597.

[25] A. Mineo, M. Palesi, G. Ascia, and V. Catania, "Runtime tunable transmitting power technique in $\mathrm{mm}$ wave winoc architectures," IEEE Transactions on Very Large Scale Integration (VLSI) Systems, vol. 24, no. 4, pp. 1535-1545, April 2016.

[26] X. Yu, H. Rashtian, S. Mirabbasi, P. P. Pande, and D. Heo, "An 18.7-gb/s 60-ghz ook demodulator in 65nm cmos for wireless network-on-chip," IEEE Transactions on Circuits and Systems I: Regular Papers, vol. 62, no. 3, pp. 799-806, March 2015.

[27] S. Deb, A. Ganguly, P. P. Pande, B. Belzer, and D. Heo, "Wireless noc as interconnection backbone for multicore chips: Promises and challenges," IEEE Journal on Emerging and Selected Topics in Circuits and Systems, vol. 2, no. 2, 2012.
[28] H. K. Mondal, R. C. Cataldo, C. A. Missio Marcon, K. Martin, S. Deb, and J. Diguet, "Broadcast- and power-aware wireless noc for barrier synchronization in parallel computing," in 2018 31st IEEE International System-on-Chip Conference (SOCC), 2018.

[29] T. Krishna, L.-S. Peh, B. M. Beckmann, and S. K. Reinhardt, "Towards the ideal on-chip fabric for 1-tomany and many-to-1 communication," in Proceedings of the 44th Annual IEEE/ACM International Symposium on Microarchitecture, ser. MICRO-44, 2011.

[30] A. K. Biswas, S. K. Nandy, and R. Narayan, "Network-on-chip router attacks and their prevention in mp-socs with multiple trusted execution environments," in 2015 IEEE International Conference on Electronics, Computing and Communication Technologies (CONECCT), July 2015, pp. 1-6.

[31] A. K. Biswas, S. K. Nandy, and R. Narayan, "Router attack toward noc-enabled mpsoc and monitoring countermeasures against such threat," Circuits, Systems, and Signal Processing, vol. 34, no. 10, pp. 32413290, Oct 2015.

[32] V. Catania, A. Mineo, S. Monteleone, M. Palesi, and D. Patti, "Improving energy efficiency in wireless network-on-chip architectures," ACM Journal on Emerging Technologies in Computing Systems, vol. 14, no. 1, 2017.

[33] T. E. Carlson, W. Heirman, S. Eyerman, I. Hur, and L. Eeckhout, "An evaluation of high-level mechanistic core models," ACM Transactions on Architecture and Code Optimization (TACO), 2014.

[34] S. C. Woo, M. Ohara, E. Torrie, J. P. Singh, and A. Gupta, "The splash-2 programs: Characterization and methodological considerations," in Proceedings of the 22Nd Annual International Symposium on Computer Architecture, ser. ISCA '95, 1995.

[35] C. Bienia, "Benchmarking modern multiprocessors," Ph.D. dissertation, Princeton University, January 2011.

[36] X. Yu, J. Baylon, P. Wettin, D. Heo, P. P. Pande, and S. Mirabbasi, "Architecture and design of multichannel millimeter-wave wireless noc," IEEE Design Test, vol. 31, no. 6, 2014.

[37] W. Huang, S. Ghosh, S. Velusamy, K. Sankaranarayanan, K. Skadron, and M. Stan, "Hotspot: a compact thermal modeling methodology for earlystage vlsi design," Very Large Scale Integration (VLSI) Systems, IEEE Transactions on, vol. 14, no. $5,2006$.

[38] A. Kahng, B. Li, L.-S. Peh, and K. Samadi, "Orion 2.0: A fast and accurate noc power and area model for early-stage design space exploration," in Design, Automation Test in Europe Conference Exhibition, 2009. DATE '09., 2009.

[39] A. K. Biswas, "Source authentication techniques for network-on-chip router configuration packets," J. Emerg. Technol. Comput. Syst., vol. 13, no. 2, Nov. 2016.

[40] "Role based shared memory access control mechanisms in noc based mp-soc," Nano Communication Networks, vol. 7. 


\section{APPENDIX A \\ Methods to calculate the Hub Connec- TION ROUTER POSITION}

As already mentioned in Section 3, each cluster has a centrally placed wireless interface connected to hub router. The hub router is connected to 4 hub connection (HC) routers. Also, a cluster (refer to Figure 1(a)) is divided into sub-clusters, where each sub-cluster consists of 4 routers (arranged in $2 \times 2$ mesh) with one HC router. Next, we present the method to calculate the position of $\mathrm{HC}$ routers. The head flit of the packet provides the source and destination router addresses. The addresses are used by Algorithms ?? and ?? to find the cluster and sub-cluster ids of the source and destination routers. The cluster and sub-cluster id of the respective routers are provided as inputs to Algorithm ?? which gives the corresponding HC router id. Please note that router ids can also be represented as router addresses where the former is an integer representation while the later is the binary representation of the same.
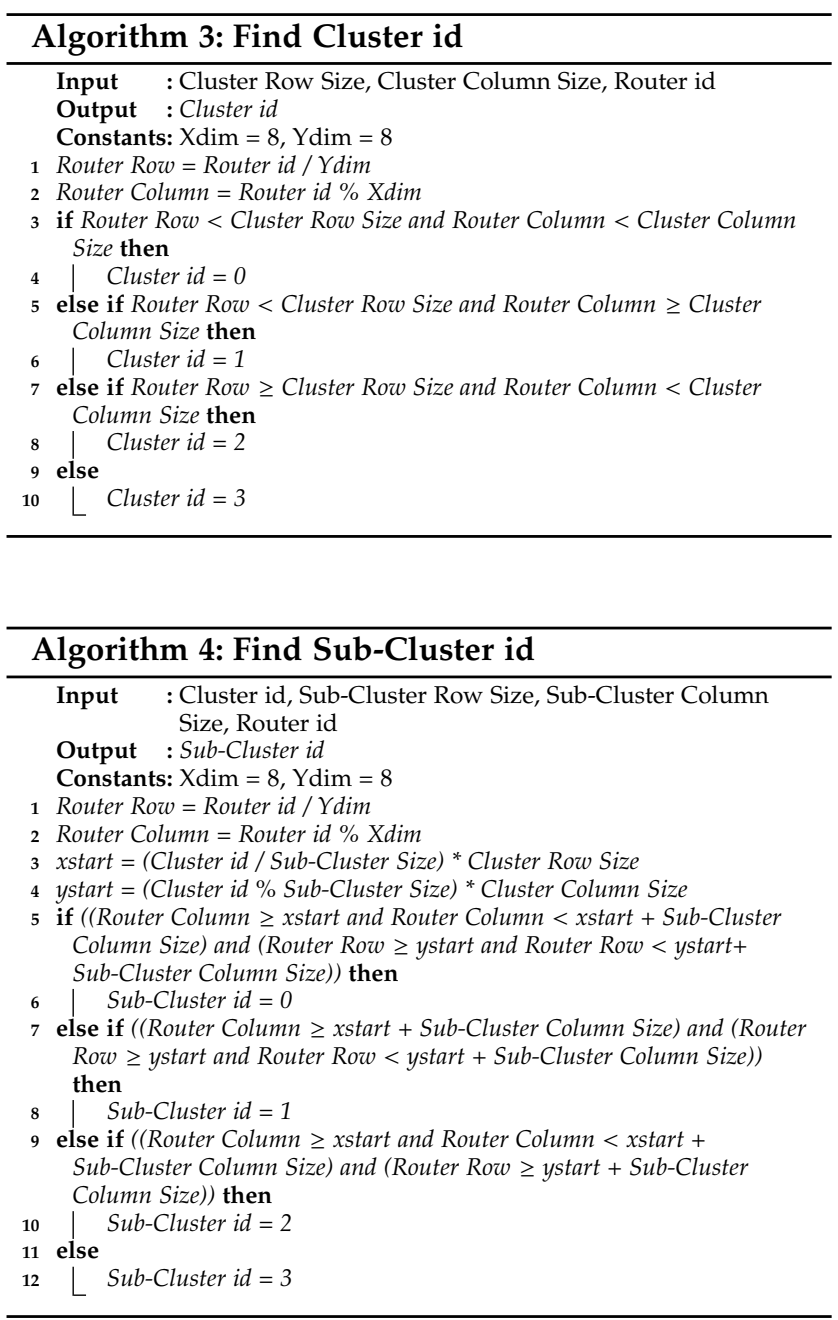

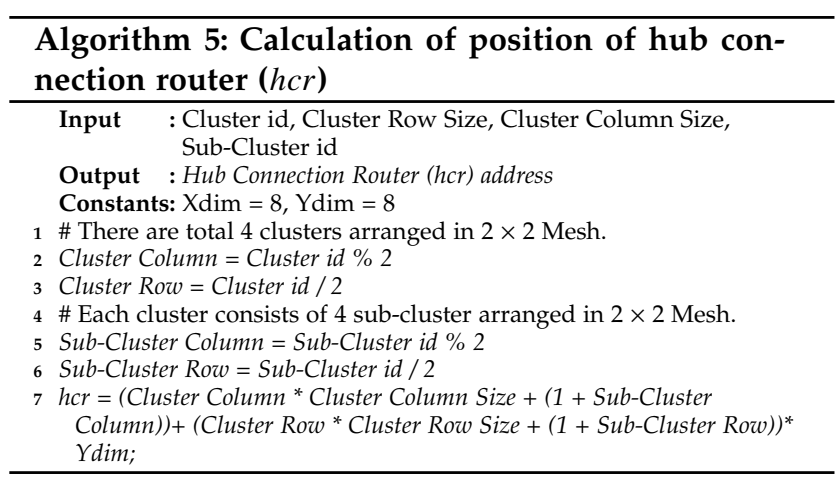

